\title{
Enhanced Radiosensitization Effect of Curcumin Delivered by PVP-PCL Nanoparticle in Lung Cancer
}

\author{
Cuixia Wen, ${ }^{1}$ Yun Zhou, ${ }^{1}$ Chong Zhou, ${ }^{1}$ Yifan Zhang, ${ }^{1}$ Xiang Hu, ${ }^{1}$ Jun Li, ${ }^{2}$ and Haitao Yin \\ ${ }^{1}$ Department of Radiotherapy, The Central Hospital of Xuzhou, Affiliated Hospital of Southeast University, Xuzhou, China \\ ${ }^{2}$ Department of Respiratory Medicine, The Central Hospital of Xuzhou, Affiliated Hospital of Southeast University, Xuzhou, China \\ Correspondence should be addressed to Jun Li; 09zhongxinyiyuan@sina.com and Haitao Yin; baxial108@126.com
}

Received 1 January 2017; Accepted 6 March 2017; Published 5 July 2017

Academic Editor: Phuong H. L. Tran

Copyright (c) 2017 Cuixia Wen et al. This is an open access article distributed under the Creative Commons Attribution License, which permits unrestricted use, distribution, and reproduction in any medium, provided the original work is properly cited.

\begin{abstract}
Curcumin, the principal polyphenolic curcuminoid, has been reported in numerous studies for its antitumor effect in a series of cancers. It is also reported that curcumin possesses radiosensitization effect in some cancers. However, the poor solubility and unsatisfied bioavailability of curcumin significantly undermine its potential application. Here we prepared curcumin loaded nanoparticles by employing PVP-PCL as drug carrier. Characterization studies indicated the satisfied drug loading efficiency and a sustained in vitro release pattern. Quantification uptake study showed that the uptake efficiency of Cum-NPs by lung cancer cells was time- and dose-dependent. In vitro anticancer study demonstrated the superior cytotoxic effect of Cum-NPs with stronger apoptotic induction over free Cum. Most importantly, there is almost no report on the radiosensitization effect of curcumin loaded nanoparticles. Here, Cum-NPs led to more inhibition of the colony forming ability of A549 cells as compared to the equivalent concentration of free Cum as shown in clonogenic assay. Furthermore, Cum-NPs are much more effective in enhancing the tumor growth inhibitory effect of radiation therapy in a A549 xenograft model. Therefore, results from the current study seem to be the first report on the radiosensitization effect of Cum-NPs and paved the way for a curcumin nanodrug delivery system as a potential radiation adjuvant.
\end{abstract}

\section{Introduction}

Curcumin (Cum), the principal polyphenolic curcuminoid, has been reported in numerous studies for its antitumor effect in a series of cancers $[1,2]$. Previous studies in the authors' lab have demonstrated the chemosensitizing effect of curcumin in lung cancer and glioma [3, 4]. However, the poor solubility and unsatisfied bioavailability of curcumin significantly undermine its potential application. Latest progress in nanotechnology, especially nanodrug delivery system, has shed the light on the improvement of curcumin delivery [5].

In our earlier studies, amphiphilic block copolymers were chosen as drug carriers due to their unique characteristics $[6,7]$. Amphiphilic copolymers consisted of a hydrophobic part and a hydrophilic part, which can self-assemble into nanoparticles in aqueous phase [8]. The hydrophobic part, such as poly(caprolactone), can effectively encapsulate insoluble drugs as inner core of the nanoparticle, while the hydrophilic segment, such as polyethylene glycol, is able to form the outer layer of the nanoparticle increasing the solubility and help the nanoparticle escape from the scavenging of the reticuloendothelial systems (RES) [9]. In previous reports, we formed curcumin loaded methoxy polyethylene glycol-poly(caprolactone) (mPEG-PCL) nanoparticles with nanoprecipitation method and demonstrated the superior anticancer effect of the drug loaded nanoparticles $[6,7]$.

One of the biggest problems of PEG is the fast clearance due to the partial activation of complement systems and the failure to fully avoid the uptake by RES [10]. To overcome this, another kind of hydrophilic polymer, poly(Nvinylpyrrolidone) (PVP), is developed as an alternative substitute for PEG. As reported previously, the modification of PVP can increase the circulation time of the nanoparticles by more efficient escape from the RES than pegylated nanoparticles [11].

Though there are a lot of studies reporting the anticancer and chemosensitization effect of nanodrug delivery systems of curcumin with amphiphilic copolymers as drug carriers 
[12-14], very few studies investigated the radiosensitization of curcumin loaded nanoparticles [15]. As reported previously, curcumin loaded PLGA nanoparticles were effective in inhibiting the growth of ovarian cancer cells and enhanced the effect of radiation. Moreover, there is no related report on the radiosensitization effect of curcumin loaded nanoparticles with PVP-PCL as drug carriers.

Here we prepared curcumin loaded nanoparticles by employing PVP-PCL as drug carrier. Detailed physiochemical characterization was performed to measure the size, zeta potential, loading efficiency, and in vitro release pattern of the drug loaded nanoparticles. SER10, calculated as the ratio of doses required to achieve $10 \%$ surviving fraction for cells, is one of the most important indicators for radiosensitization evaluation. The values of SER10 were calculated from the curves of clonogenic assay. The enhanced in vitro and in vivo radiosensitization effect and possible underlying mechanism were reported for the first time, which solidified the promising application of curcumin loaded PVP-PCL nanoparticles as a novel radiosensitizer.

\section{Materials and Methods}

2.1. Materials. Curcumin was purchased from Sigma Aldrich Co. Ltd (St. Louis, MO, USA). PVP-PCL was kindly provided by Dr. Xiaolin Li from Nanjing Medical University. Human lung cancer cell line A549 was obtained from Institute of Biochemistry and Cell Biology, Chinese Academy of Sciences (Shanghai, China). Male and female nude mice (nu/nu; 6-8 weeks old and weighing 18-22 g) were maintained in the animal facility of the Animal Center of Xuzhou Normal University. The animal protocol was reviewed and approved by the Institutional Animal Care and Use Committee of Xuzhou Normal University.

\subsection{Methods}

2.2.1. Preparation of Curcumin Loaded Nanoparticles. Curcumin loaded nanoparticles were constructed as previously reported by us [6]. Briefly, $20 \mathrm{mg}$ of PVP-PCL and $4 \mathrm{mg}$ Cum were slowly dissolved in $500 \mathrm{ul}$ acetone. Then the solution was added drop by drop into $20 \mathrm{~mL}$ double distilled water with gentle agitation at room temperature. The mixed solution was transferred into a dialysis bag with a cut-off molecular weight of $12 \mathrm{kd}$ and dialyzed for $8 \mathrm{~h}$ with water replacement every $1 \mathrm{~h}$. The solution was then filtered through a $220 \mathrm{~nm}$ filter membrane to remove aggregates and freeze-dried for future use.

2.2.2. Characterization and In Vitro Release Pattern of Curcumin Loaded Nanoparticles. Dynamic light scattering and zeta potential analyzer (Brookhaven Instruments Corporation) were used to measure the size and zeta potential, respectively. The concentration of Cum was detected by a HPLC system (Shimadzu, Tokyo, Japan). The mobile phase consisted of acetonitrile-monosodium phosphate $(10 \mathrm{mM}$, pH 3.5 adjusted by orthophosphoric acid) $(50: 50, \mathrm{v} / \mathrm{v})$ at a flow rate of $1.0 \mathrm{ml} / \mathrm{min}$ with a detecting wavelength of $425 \mathrm{~nm}$.
The following equations were adopted to calculate the drug loading content and encapsulation efficiency of Cum-NPs.

In vitro release study was performed by a method of dialysis [13]. Briefly, $10 \mathrm{mg}$ of freeze-dried Cum-NPs was dissolved in $1 \mathrm{ml}$ double distilled water and put into a dialysis bag. The dialysis bag was immersed into $50 \mathrm{~mL}$ PBS with gentle agitation at room temperature. At designated time points, $1 \mathrm{~mL}$ PBS solution outside the bag was taken out for HPLC measurement of Cum concentration. Immediately the equivalent volume of fresh PBS was supplemented into the incubation medium. The percent of total Cum released from the nanoparticles was calculated and plotted as a function of time.

2.2.3. The Uptake Efficiency of Cum-NPs by Cancer Cells. As reported in our previous study, uptake efficiency was quantified by HPLC [16]. Cum-NPs at a concentration of $20 \mathrm{uM}$ were incubated with A549 cells for either 2 or $4 \mathrm{~h}$. After the incubation, cells were washed, digested by trypsin, and collected by centrifuge. The cell pellets were then dissolved in methanol. Cum was extracted through sonication of the methanol solution and subjected to HPLC measurement.

2.2.4. In Vitro Cytotoxicity Test. In vitro cytotoxicity was tested by MTT assay as reported previously [6]. Briefly, cells were seeded in a 96-well plate at a density of 5000-10000 cells per well. Cells were treated with escalated doses of Cum and Cum-NPs at equivalent doses ranging from 5 to $80 \mathrm{uM}$ and incubated for 24 or $48 \mathrm{~h}$, respectively. After the incubation, cells were washed and $20 \mu \mathrm{l}$ of $5 \mathrm{mg} / \mathrm{ml}$ MTT solution was added to each well for another incubation of $4 \mathrm{~h}$. After that, the medium was discarded and 150 ul DMSO was added to each well. Then the optical density of each well was detected by a microplate reader (Bio-Rad, Hercules, USA) at a wavelength of $560 \mathrm{~nm}$.

2.2.5. FACS Analysis. A549 cells were treated with the equivalent concentration of Cum and Cum-NPs at $20 \mathrm{uM}$ for $24 \mathrm{~h}$ followed by irradiation with $2 \mathrm{~Gy}$. Cells were then stained by annexin V-FITC and propidium iodide (PI) with an annexin V-FITC kit and subjected to analysis by a FACScan flow cytometer (Becton Dickinson, CA, USA).

2.2.6. Clonogenic Assay. Clonogenic assay was performed to evaluate the radiosensitization effect of Cum-NPs. Briefly, A549 cells were seeded into Petri dishes at a density of $70-80 \%$ confluency. The cells were treated with the equivalent concentration of Cum and Cum-NPs at $20 \mathrm{uM}$ for $4 \mathrm{~h}$ followed by irradiation with different doses $(2,4$, and $6 \mathrm{~Gy})$. Cells were precisely tuned to predetermined densities immediately after irradiation and seeded in 6-well plate at different densities. After 2 weeks of incubation, the plates were dyed by $0.5 \%$ crystal violet and the colonies were counted manually to plot survival curve.

2.2.7. In Vivo Evaluation of the Radiosensitization Effect of Cum-NPs. Nude mice (6-8 weeks) were raised in a SPF environment. 200 ul cell suspension with $1 * 10^{6}$ A549 cells 
TABLE 1: Size and load efficiency of Cum-NPs.

\begin{tabular}{lccccc}
\hline Nanoparticles & Particle size $(\mathrm{nm})^{\mathrm{a}}$ & Polydispersity & Zeta potential $(\mathrm{mV})$ & ${\text { DLC }(\%)^{\mathrm{b}}}$ & EE $(\%)^{\mathrm{c}}$ \\
\hline Cum-NPs & $95.7 \pm 5.6$ & $0.13 \pm 0.03$ & $-3.3 \pm 1.1$ & $12.6 \pm 3.2$ & $83.2 \pm 3.6$ \\
\hline
\end{tabular}

${ }^{\mathrm{a}}$ The SD value was for the mean particle size obtained from the three measurements of a single batch; ${ }^{\mathrm{b}} \mathrm{DLC}=$ drug loading content; ${ }^{\mathrm{c}} \mathrm{EE}=\mathrm{encapsulation}$ efficiency.

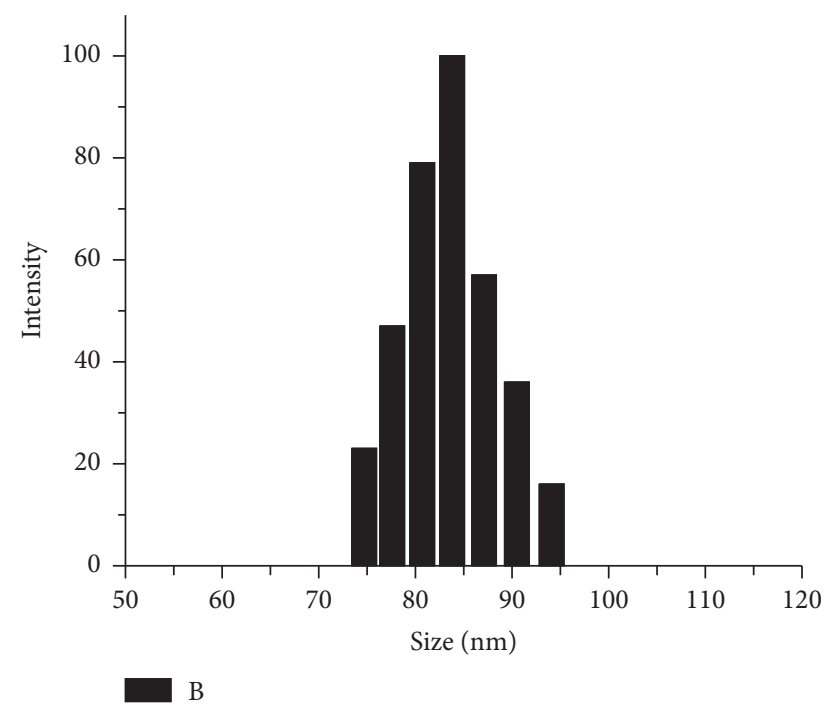

FIGURE 1: Size distribution of Cum-NPs by DLS.

was injected subcutaneously in left axillary space of mice. Treatments were started after 7-10 days of implantation. Day 0 was designated as the day when the tumor volume reached $100 \mathrm{~mm} 3$. The mice were then randomly divided into six groups with 6 mice per group. Treatments included the following regimens: Group 1: control group; Group 2: $20 \mathrm{mg} / \mathrm{kg}$ Cum by i.v. injection on Day 1: Group 3: $20 \mathrm{mg} / \mathrm{kg}$ Cum-NPs by i.v. injection on Day 1; Group 4: irradiation at a dose of $8 \mathrm{~Gy}$ on Day 2; Group 5: $20 \mathrm{mg} / \mathrm{kg}$ Cum by i.v. injection on Day 1 followed by irradiation at a dose of 8 Gy on Day 2; Group 6: $20 \mathrm{mg} / \mathrm{kg}$ Cum-NPs by i.v. injection on Day 1 followed by irradiation at a dose of 8 Gy on Day 2. Tumors were measured every 2 days with calipers till the end of the experiment. Tumor volume was calculated by the equation $\left(W^{2} * L\right) / 2$, where $W$ is the tumor measurement at the widest point and $L$ is the tumor dimension at the longest point. Relative Tumor Volume (RTV) was calculated by the equation $(V n / V 0)$, where $V n$ is the tumor volume measured at the corresponding day and $V 0$ is the tumor volume measured at Day 0.

2.2.8. Statistical Analysis. Data were expressed as the mean \pm SD. Student's $t$-test and analysis of variance were used to analyze the data. $p<0.05$ was considered statistically significant.

\section{Results and Discussion}

3.1. Characterization of Curcumin Loaded Nanoparticles (Cum-NPs). As shown in Figure 1 and Table 1, the mean

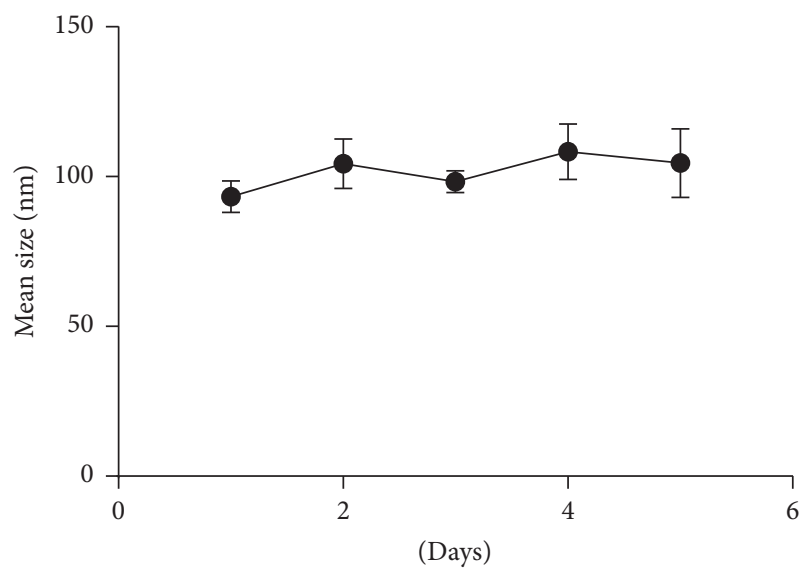

Figure 2: Size change of Cum-NPs at room temperature in 5 days.

size of Cum-NPs was $95.7 \pm 5.6 \mathrm{~nm}$ with a polydispersity of 0.13 , which indicated relatively narrow size distribution. To investigate the stability of Cum-NPs, continuous size change was measured by DLS. The size of Cum-NPs showed no obvious change during the observation of 5 days after preparation in routine temperature demonstrating satisfied stability (Figure 2). In addition, zeta potential of Cum-NPs was around $-3.3 \pm 1.1 \mathrm{mV}$ (Table 1 ). It is reported in previous studies that the outer shell by PVP modification of the nanoparticles could neutralize the surface charge to certain extent, which is in accordance with the current results [17].

Table 1 showed the loading efficiency of Cum-NPs. The highest drug loading content was $12.6 \pm 3.2 \%$ with the encapsulation efficiency being more than $83 \%$. As reported in previous studies, the good affinity between the lipophilic PCL core and the hydrophobic curcumin enables the high drug loading efficiency, while the good biocompatibility of PVP leads to the excellent stability of the whole particle $[17,18]$.

During the in vitro release study, curcumin was released from PVP-PCL nanoparticles in a sustained manner with certain initial burst release (Figure 3). Less than $30 \%$ of total amount was detected in the outer medium, whereas the other $30 \%$ of drugs were released in the last 5 days. The last $35 \%$ of curcumin still remained in the nanoparticles at the end of the experiment. The burst release was attributed to the drug attached to the surface of nanoparticles while the sustained release was achieved by the affinity between the encapsulated curcumin and hydrophobic PCL core [15]. Thus, data from the figure indicated Cum-NPs as a promising controlled release system for cancer therapy.

3.2. Cellular Uptake of Cum-NPs. Quantification analysis showed that the uptake efficiency of Cum-NPs by cancer cells was time- and dose-dependent (Figure 4). In detail, 


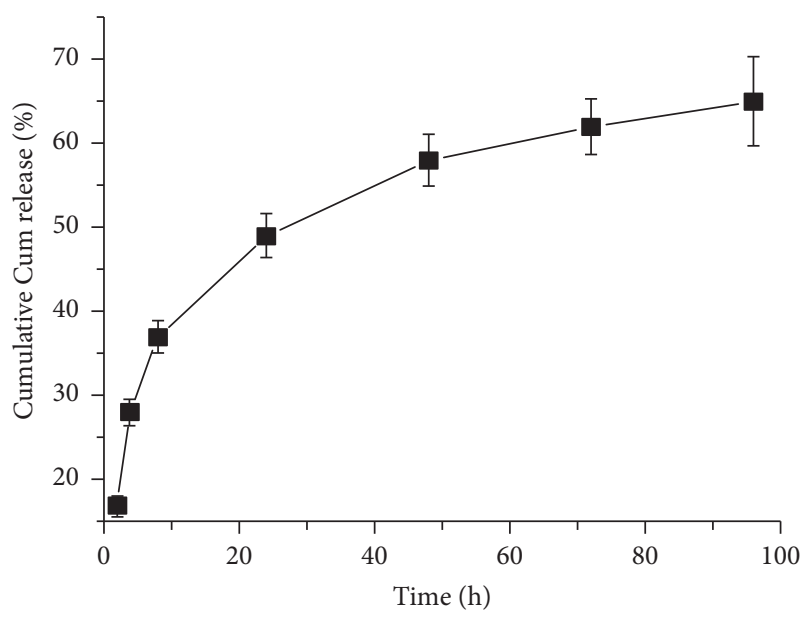

FIGURE 3: In vitro release curve of Cum-NPs.

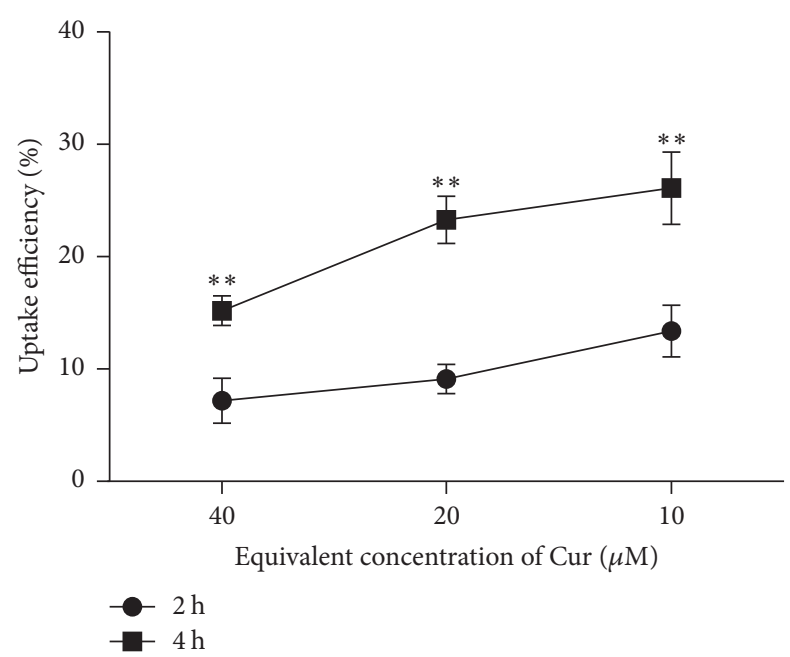

Figure 4: Cellular uptake efficiency of Cum-NPs by A549 cells. ** represents $p<0.01$ versus the corresponding group.

extending of incubation time significantly almost doubled the internalization of Cum-NPs. On the contrary, increase of the concentration led to the decrease of uptake. According to previous studies, the efficiency of cellular nanoparticle uptake mainly depends on the penetration of cell membrane, including a special process called endocytosis, which is much more efficient than the filtration of small molecules [18]. During this process, incubation time seems to be one of the main factors with higher efficiency achieved by extending the period. In addition, another parameter is the concentration of the encapsulated drug. As reported here and in earlier studies, there is a limit for endocytosis and the uptake efficiency will go higher if the concentration is much more higher than the saturable ability of endocytosis [19].

3.3. In Vitro Cytotoxicity of Cum-NPs against Lung Cancer. The in vitro cytotoxicity of Cum-NPs against A549 cells was measured by XTT assay (Figure 5). First, it is noted that the empty polymeric nanoparticles had no obvious toxicity to the cells even at the highest concentration of $500 \mathrm{ug} / \mathrm{ml}$. Both

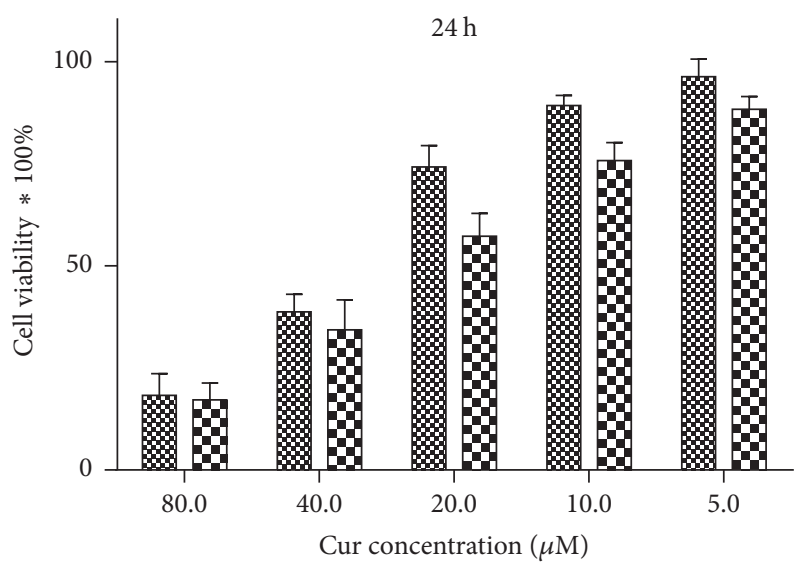

\%) Cur

Q0ur-NPs

(a)

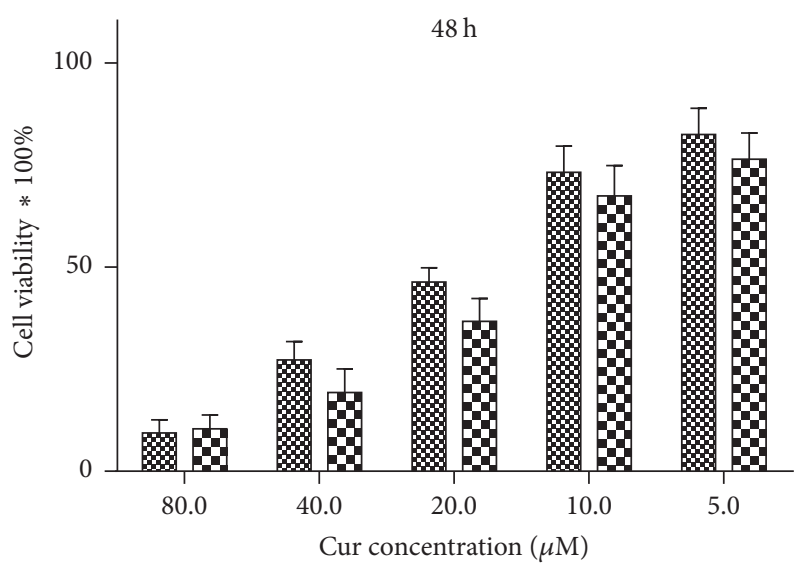

\%) Cur

Dur-NPs

(b)

FIGURE 5: In vitro cytotoxicity of Cum-NPs and free Cum against A549 cells for $24 \mathrm{~h} \mathrm{(a)} \mathrm{and} 48 \mathrm{~h} \mathrm{(b).}$

free curcumin and Cum-NPs time- and dose-dependently inhibited the proliferation of A549 cells. Moreover, CumNPs seem to induce more cell death than free curcumin at relatively lower equivalent concentrations. For example, about $74 \%$ of cells remained alive after being treated by $20 \mathrm{uM}$ free curcumin for $24 \mathrm{~h}$ while less than $60 \%$ of cells survived under the treatment of the equivalent concentration of CumNPs. However, extending of the incubation time tends to ameliorate the discrepancy. After $48 \mathrm{~h}$ incubation, there were about $46 \%$ of A549 cells left alive with the same dose of CumNPs only leading to $10 \%$ more cell death.

Reasons for the discrepancy of the cytotoxicity of curcumin and Cum-NPs may result from the different uptake efficiency [19]. As shown in the uptake study, nanoparticle delivery was more efficient in penetrating the membrane by endocytosis than the filtration of small drug molecules, thereby enabling more drug accumulation in cancer cells, which may be the basis for the discrepancy at lower concentrations $[13,20]$. Nevertheless, if the concentration was higher 


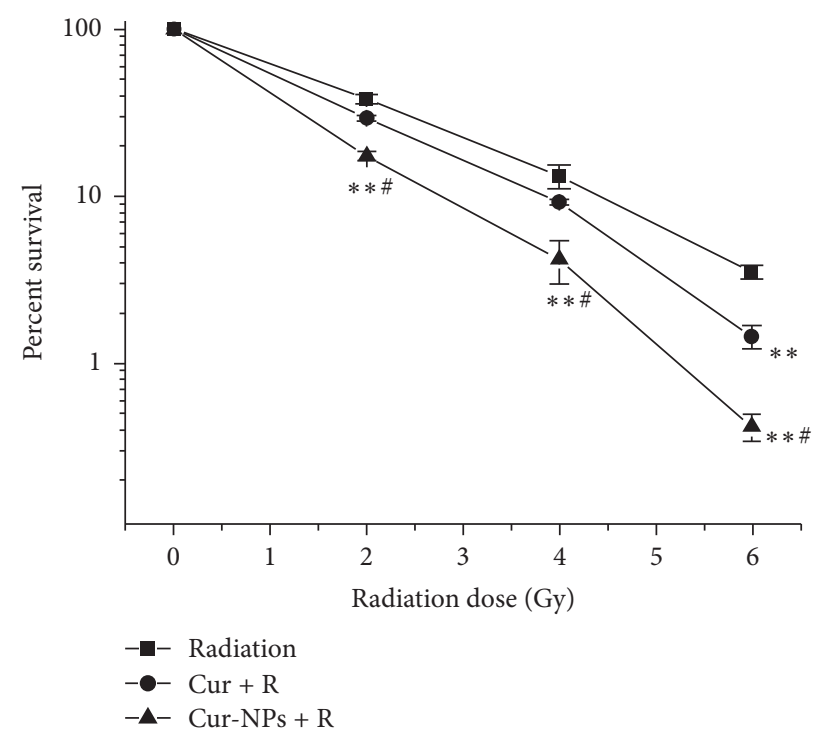

FIGURE 6: In vitro radiosensitization effects of Cum-NPs and free Cum by clonogenic assay. $* *$ represents $p<0.01$ versus radiation group. \# represents $p<0.05$ versus the group receiving free Cum plus radiation.

than the limit of endocytosis, the internalization would not increase as well, which was in accordance with the lower discrepancy of cytotoxicity between Cum-NPs and curcumin at higher doses [21].

3.4. Radiosensitization Effect of Cum-NPs against Lung Cancer. As shown in Figure 6, clonogenic assay indicated that both free curcumin and Cum-NPs sensitized A549 cells to radiation. Most importantly, Cum-NPs led to more inhibition of the colony forming ability of A549 cells as compared to the equivalent concentration of free curcumin. In detail, the significant difference was identified only between the group treated singly or in combination with free curcumin and that treated with $6 \mathrm{~Gy}$ radiation instead of the other two doses of radiation ( 2 and $4 \mathrm{~Gy}$ ). On the contrary, there was a significant difference between singly or combinational administration of Cum-NPs and either dose of radiation, demonstrating the superior radiosensitization effect of nanoparticle delivery of curcumin.

SER10 was calculated from the clonogenic curve. The SER10 of free curcumin in A549 cells was 1.13, which is substantially smaller than that of Cum-NPs at 1.55. As reported from previous studies, the SER10 of free curcumin in different cancer cells was from 1.1 to 1.4 [22]. Here we demonstrated that the delivery of curcumin by PVP-PCL nanoparticles significantly increased the sensitivity of cancer cells to radiation therapy, thereby paving the way for a curcumin nanodrug delivery system as a potential radiation adjuvant.

3.5. Apoptotic Induction of the Radiosensitization Effect of Cum-NPs. FACS was utilized to evaluate the apoptotic inducing effect of different treatment regimens (Figure 7). As shown in the figure, Cum-NPs led to more early and late apoptosis than free Cum did at the equivalent dose. Radiation alone had stronger apoptotic induction than either Cum or Cum-NPs. Moreover, sequential administration of CumNPs and irradiation generated the most significant cellular apoptosis with the lowest survival among all the groups, demonstrating the superior radiosensitization effect of CumNPs over free Cum.

As reported in previous studies, Cum can effectively sensitize a series of tumors to both chemotherapy and radiotherapy [23-25]. However, few studies have studied the apoptotic inducing effect of curcumin loaded nanoparticles together with irradiation, especially PVP-PCL nanoparticles. The current study reported for the first time that Cum-NPs, in combination with irradiation, could effectively lead to much more early and late apoptosis with lower cell survival than free Cum did.

3.6. In Vivo Radiosensitization Effect of Cum-NPs. A549 xenograft model was established to investigate the radiosensitization effect of Cum-NPs (Figure 8). First, the tumor nodule in curcumin treated group grew much slower than that in Cum-NPs treated group although there was a significant difference of RTV between the control group and either of the treatment groups. Second, the combination of radiation and either curcumin or Cum-NPs produced a much more inhibitory effect than each of the single treatments. Moreover, when combined with radiation, RTV from the group of Cum-NPs was significantly lower than the group treated with the equivalent dose of curcumin, which demonstrated the enhanced radiosensitization effect of Cum-NPs in A549 xenograft model. For example, RTV of the group treated by radiation was 398 , which was significantly smaller than either of the nonradiation drug-treated groups. Cum-NPs plus radiation achieved a RTV value of 224 while RTV of the curcumin plus radiation treated group was 334. Statistical analysis revealed that there was a significant difference of RTV between the two groups treated with radiation alone and Cum-NPs plus radiation. However, no significant difference in RTV was found between the curcumin treated group and curcumin plus radiation treated group. In addition, there was no significant difference in the activity or behavior of the animals between the groups receiving Cum-NPs and Cum, which demonstrated no obvious toxicity of Cum-NPs when delivered in vivo.

A lot of earlier studies report the chemosensitization and radiosensitization effect of curcumin in a series of cancers by elucidating the possible underlying mechanisms [26-28]. Recently increasing evidences show the potential of nanodrug delivery systems in improving the pharmacodynamic and pharmacokinetics profile of curcumin [29-31]. In the author's previous studies, amphiphilic block polymers were employed as drug carriers [6]. However, previous studies including ours mainly focus on the chemosensitization effect of curcumin loaded nanoparticles [32, 33]. Very few reports investigate the radiosensitization effect of curcumin loaded nanoparticles. Hence, this study seems to be the first report to demonstrate the superior radiosensitization effect of curcumin loaded PVP-PCL nanoparticles. The following study is ongoing in the author's lab to examine the possible 

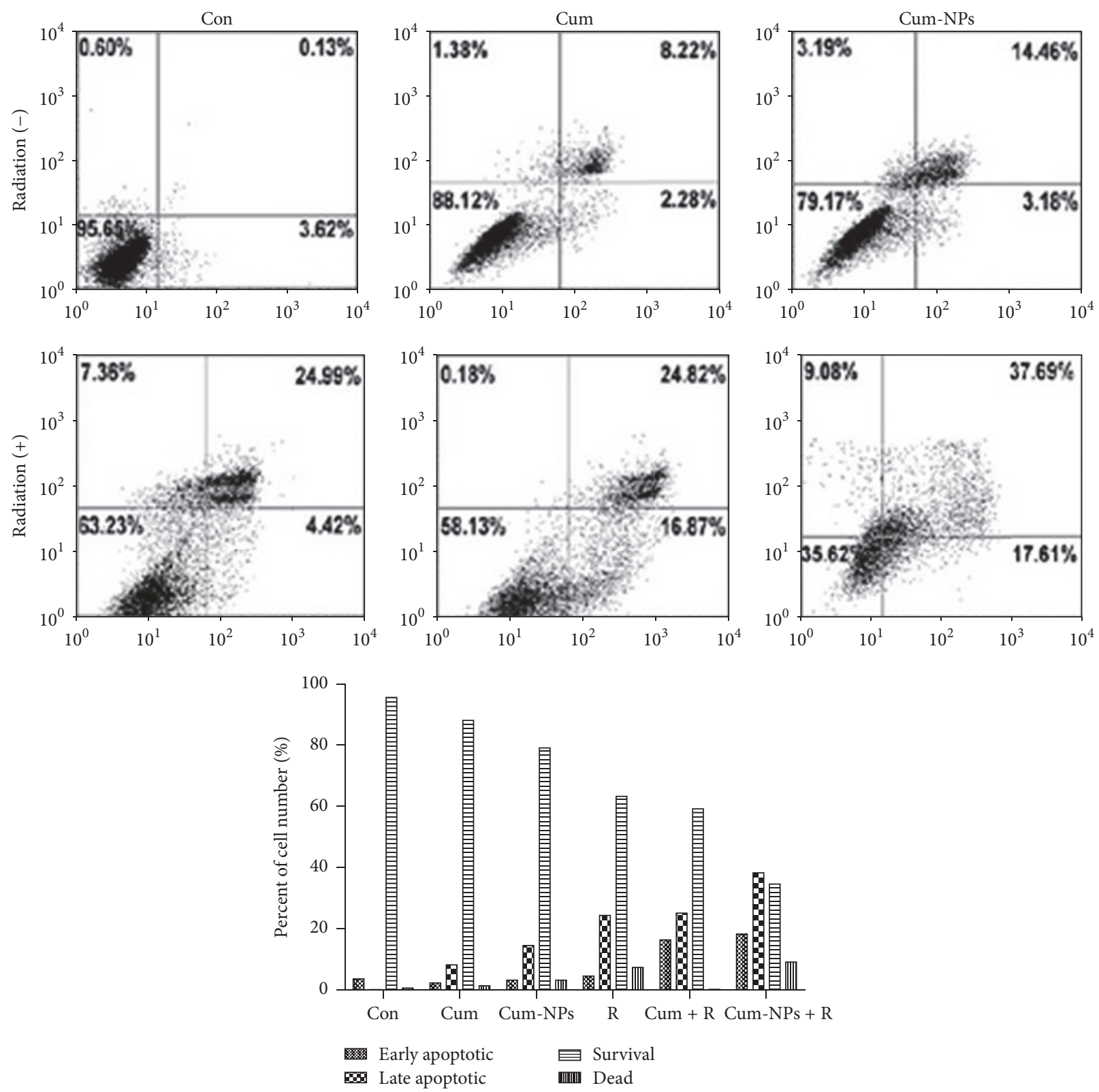

Figure 7: Apoptotic induction of Cum-NPs and free Cum with or without the combination of radiation in A549 cells.

mechanisms. Planned modification is still under productive consideration to improve the loading efficiency and targetability of the drug loaded nanoparticles. Dual drug delivery systems based on the synergistic radiosensitization are also a novel direction to achieve better therapeutic efficacy with radiation.

\section{Conclusion}

In the current study, an amphiphilic block copolymer PVPPCL was synthesized and employed as drug carrier. Curcumin was encapsulated into PVP-PCL nanoparticles to form a feasible nanodrug delivery system. Physiochemical characterization showed the stability of Cum-NPs with a satisfied drug loading efficiency and sustained release pattern. In vitro study demonstrated that Cum-NPs inhibited the proliferation of cancer cells by inducing apoptosis more efficiently than the equivalent dose of free curcumin. Moreover, Cum-NPs were much more effective in enhancing the sensitivity of cancer cells to radiation from the results of in vitro clonogenic assay and in vivo tumor growth evaluation. Therefore, it is valuable to perform extensive research on the potential application of the nanodelivery system of curcumin as a novel nanoradiosensitizer.

\section{Conflicts of Interest}

The authors report no conflicts of interest in this work. 


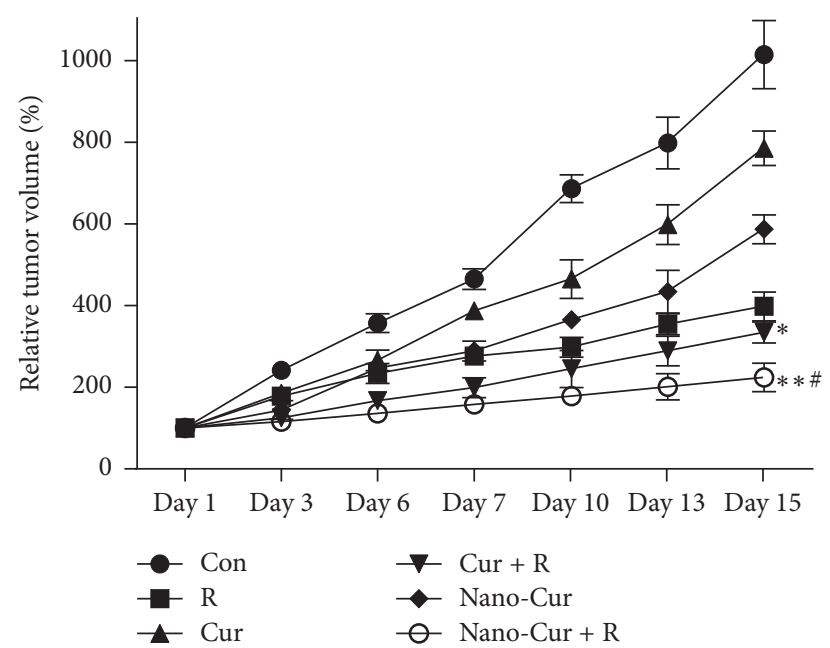

Figure 8: In vivo radiosensitization effect of Cum-NPs and free Cum in a xenograft model of A549 cells. * represents $p<0.05$ versus radiation group. $* *$ represents $p<0.01$ versus radiation group. \# represents $p<0.05$ versus the group receiving free Cum plus radiation.

\section{Authors' Contributions}

Cuixia Wen and Yun Zhou contributed equally to this work.

\section{Acknowledgments}

This work was supported by the National Natural Science Foundation of China (no. 81672973) and Natural Science Foundation of Jiangsu Province (BK20151157).

\section{References}

[1] J.-A. Seo, B. Kim, D. N. Dhanasekaran, B. K. Tsang, and Y. S. Song, "Curcumin induces apoptosis by inhibiting sarco/endoplasmic reticulum Ca2+ ATPase activity in ovarian cancer cells," Cancer Letters, vol. 371, no. 1, pp. 30-37, 2016.

[2] C. Rana, H. Piplani, V. Vaish, B. Nehru, and S. N. Sanyal, "Downregulation of telomerase activity by diclofenac and curcumin is associated with cell cycle arrest and induction of apoptosis in colon cancer," Tumor Biology, vol. 36, no. 8, pp. 5999-6010, 2015.

[3] H. Yin, Y. Zhou, C. Wen et al., "Curcumin sensitizes glioblastoma to temozolomide by simultaneously generating ROS and disrupting AKT/mTOR signaling," Oncology Reports, vol. 32, no. 4, pp. 1610-1616, 2014.

[4] H. Yin, R. Guo, Y. Xu et al., "Synergistic antitumor efficiency of docetaxel and curcumin against lung cancer," Acta Biochimica et Biophysica Sinica, vol. 44, no. 2, pp. 147-153, 2012.

[5] Y. Huang, S. P. C. Cole, T. Cai, and Y. Cai, "Applications of nanoparticle drug delivery systems for the reversal of multidrug resistance in cancer," Oncology Letters, vol. 12, no. 1, pp. 11-15, 2016.

[6] H. Yin, H. Zhang, and B. Liu, "Superior anticancer efficacy of curcumin-loaded nanoparticles against lung cancer," Acta Biochimica et Biophysica Sinica, vol. 45, no. 8, pp. 634-640, 2013.
[7] H.-T. Yin, D.-G. Zhang, X.-L. Wu, X.-E. Huang, and G. Chen, "In vivo evaluation of curcumin-loaded nanoparticles in a A549 xenograft mice model," Asian Pacific Journal of Cancer Prevention, vol. 14, no. 1, pp. 409-412, 2013.

[8] X. Li, R. Li, X. Qian et al., "Superior antitumor efficiency of cisplatin-loaded nanoparticles by intratumoral delivery with decreased tumor metabolism rate," European Journal of Pharmaceutics and Biopharmaceutics, vol. 70, no. 3, pp. 726-734, 2008.

[9] H. Kheiri Manjili, P. Ghasemi, H. Malvandi, M. S. Mousavi, E. Attari, and H. Danafar, "Pharmacokinetics and in vivo delivery of curcumin by copolymeric mPEG-PCL micelles," European Journal of Pharmaceutics and Biopharmaceutics, vol. 116, pp. 1730, 2017.

[10] T. Ishida, M. Ichihara, X. Wang et al., "Injection of PEGylated liposomes in rats elicits PEG-specific IgM, which is responsible for rapid elimination of a second dose of PEGylated liposomes," Journal of Controlled Release, vol. 112, no. 1, pp. 15-25, 2006.

[11] Y. Kaneda, Y. Tsutsumi, Y. Yoshioka et al., "The use of PVP as a polymeric carrier to improve the plasma half-life of drugs," Biomaterials, vol. 25, no. 16, pp. 3259-3266, 2004.

[12] N. M. Khalil, T. C. F. do Nascimento, D. M. Casa et al., "Pharmacokinetics of curcumin-loaded PLGA and PLGA-PEG blend nanoparticles after oral administration in rats," Colloids and Surfaces B: Biointerfaces, vol. 101, pp. 353-360, 2013.

[13] F. S. T. Mirakabad, A. Akbarzadeh, M. Milani et al., "A Comparison between the cytotoxic effects of pure curcumin and curcumin-loaded PLGA-PEG nanoparticles on the MCF7 human breast cancer cell line," Artificial Cells, Nanomedicine and Biotechnology, vol. 44, no. 1, pp. 423-430, 2016.

[14] Q. Guo, X. Li, Y. Yang et al., "Enhanced $4 \mathrm{T1}$ breast carcinoma anticancer activity by co-delivery of doxorubicin and curcumin with core-shell drug-carrier based on heparin modified poly(llactide) grafted polyethylenimine cationic nanoparticles," Journal of Biomedical Nanotechnology, vol. 10, no. 2, pp. 227-237, 2014.

[15] M. M. Yallapu, D. M. Maher, V. Sundram, M. C. Bell, M. Jaggi, and S. C. Chauhan, "Curcumin induces chemo/radiosensitization in ovarian cancer cells and curcumin nanoparticles inhibit ovarian cancer cell growth," Journal of Ovarian Research, vol. 3, no. 1, article 11, 2010.

[16] X. Li, X. Lu, H. Xu et al., "Paclitaxel/tetrandrine coloaded nanoparticles effectively promote the apoptosis of gastric cancer cells based on 'oxidation therapy"', Molecular Pharmaceutics, vol. 9, no. 2, pp. 222-229, 2012.

[17] Z. Zhu, Y. Li, X. Li et al., "Paclitaxel-loaded poly $(N-$ vinylpyrrolidone)- $b$-poly( $\varepsilon$-caprolactone) nanoparticles: preparation and antitumor activity in vivo," Journal of Controlled Release, vol. 142, no. 3, pp. 438-446, 2010.

[18] H. Xu, Z. Hou, H. Zhang et al., "An efficient Trojan delivery of tetrandrine by poly(N-vinylpyrrolidone)-block-poly(epsiloncaprolactone) (PVP-b-PCL) nanoparticles shows enhanced apoptotic induction of lung cancer cells and inhibition of its migration and invasion," International Journal of Nanomedicine, vol. 9, no. 1, pp. 231-242, 2014.

[19] X. Li, D. Zhen, X. Lu et al., "Enhanced cytotoxicity and activation of ROS-dependent c-Jun NH 2-terminal kinase and caspase- 3 by low doses of tetrandrine-loaded nanoparticles in Lovo cells-a possible Trojan strategy against cancer," European Journal of Pharmaceutics and Biopharmaceutics, vol. 75, no. 3, pp. 334-340, 2010. 
[20] B. Yameen, W. I. Choi, C. Vilos, A. Swami, J. Shi, and O. C. Farokhzad, "Insight into nanoparticle cellular uptake and intracellular targeting," Journal of Controlled Release, vol. 190, pp. 485-499, 2014.

[21] F. M. Mickler, L. Möckl, N. Ruthardt, M. Ogris, E. Wagner, and C. Bräuchle, "Tuning nanoparticle uptake: Live-cell imaging reveals two distinct endocytosis mechanisms mediated by natural and artificial EGFR targeting ligand," Nano Letters, vol. 12, no. 7, pp. 3417-3423, 2012.

[22] S. K. Sandur, A. Deorukhkar, M. K. Pandey et al., "Curcumin modulates the radiosensitivity of colorectal cancer cells by suppressing constitutive and inducible NF- $\kappa \mathrm{B}$ activity," International Journal of Radiation Oncology Biology Physics, vol. 75, no. 2, pp. 534-542, 2009.

[23] H. Fan, M. Shao, S. Huang et al., "MiR-593 mediates curcumininduced radiosensitization of nasopharyngeal carcinoma cells via MDR1," Oncology Letters, vol. 11, no. 6, pp. 3729-3734, 2016.

[24] H. J. Kang, S. H. Lee, J. E. Price, and L. S. Kim, "Curcumin suppresses the paclitaxel-induced nuclear factor-kappaB in breast cancer cells and potentiates the growth inhibitory effect of paclitaxel in a breast cancer nude mice model," The Breast Journal, vol. 15, no. 3, pp. 223-229, 2009.

[25] J. M. Hong, C. S. Park, I. S. Nam-Goong et al., "Curcumin enhances docetaxel-induced apoptosis of $8505 \mathrm{C}$ anaplastic thyroid carcinoma cells," Endocrinology and Metabolism, vol. 29, no. 1, pp. 54-61, 2014.

[26] Y.-D. Yan, N. Marasini, Y. K. Choi et al., "Effect of dose and dosage interval on the oral bioavailability of docetaxel in combination with a curcumin self-emulsifying drug delivery system (SEDDS)," European Journal of Drug Metabolism and Pharmacokinetics, vol. 37, no. 3, pp. 217-224, 2012.

[27] A. B. Kunnumakkara, P. Diagaradjane, P. Anand et al., "Curcumin sensitizes human colorectal cancer to capecitabine by modulation of cyclin D1, COX-2, MMP-9, VEGF and CXCR4 expression in an orthotopic mouse model," International Journal of Cancer, vol. 125, no. 9, pp. 2187-2197, 2009.

[28] A. B. Kunnumakkara, P. Diagaradjane, S. Guha et al., "Curcumin sensitizes human colorectal cancer xenografts in nude mice to $\gamma$-radiation by targeting nuclear factor- $\kappa \mathrm{B}$-regulated gene products," Clinical Cancer Research, vol. 14, no. 7, pp. 21282136, 2008.

[29] J.-R. Peng and Z.-Y. Qian, "Drug delivery systems for overcoming the bioavailability of curcumin: Not only the nanoparticle matters," Nanomedicine, vol. 9, no. 6, pp. 747-750, 2014.

[30] K. J. Lim, S. Bisht, E. E. Bar, A. Maitra, and C. G. Eberhart, "A polymeric nanoparticle formulation of curcumin inhibits growth, clonogenicity and stem-like fraction in malignant brain tumors," Cancer Biology and Therapy, vol. 11, no. 5, pp. 464-473, 2011.

[31] S. Bisht, G. Feldmann, S. Soni, R. Ravi, C. Karikar, and A. Maitra, "Polymeric nanoparticle-encapsulated curcumin ("nanocurcumin"): a novel strategy for human cancer therapy," Journal of Nanobiotechnology, vol. 5, article 3, 2007.

[32] B. Hu, D. Sun, C. Sun et al., "A polymeric nanoparticle formulation of curcumin in combination with sorafenib synergistically inhibits tumor growth and metastasis in an orthotopic model of human hepatocellular carcinoma," Biochemical and Biophysical Research Communications, vol. 468, no. 4, pp. 525-532, 2015.

[33] X. Kang, C. Zhao, L. Yan, R. Qi, X. Jing, and Z. Wang, "Sensitizing nanoparticle based platinum(IV) drugs by curcumin for better chemotherapy," Colloids and Surfaces B: Biointerfaces, vol. 145, pp. 812-819, 2016. 

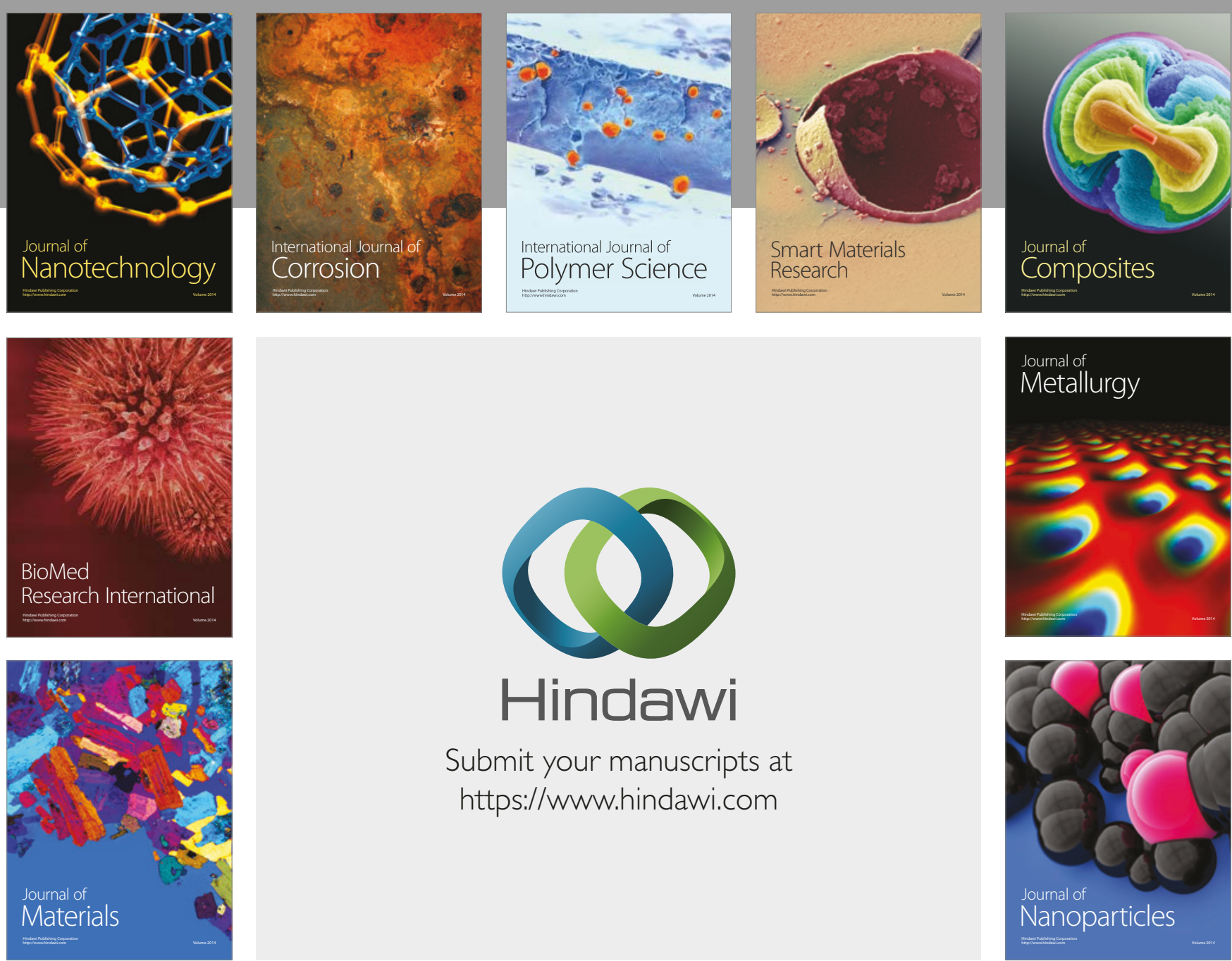

\section{Hindawi}

Submit your manuscripts at

https://www.hindawi.com
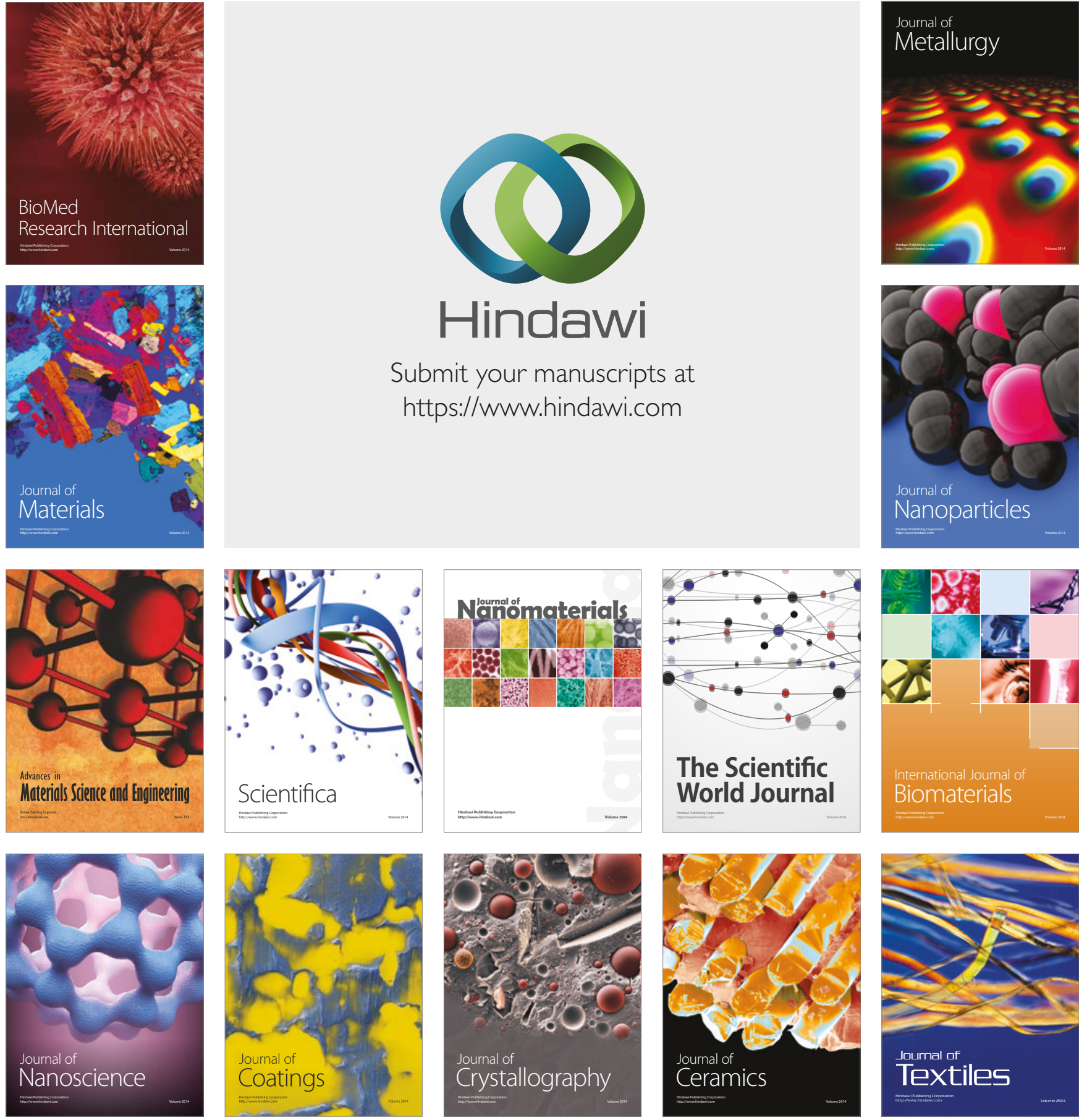

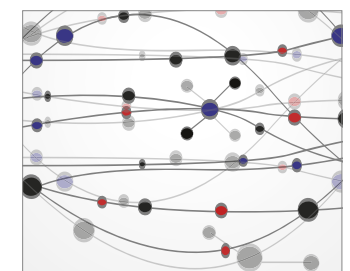

The Scientific World Journal
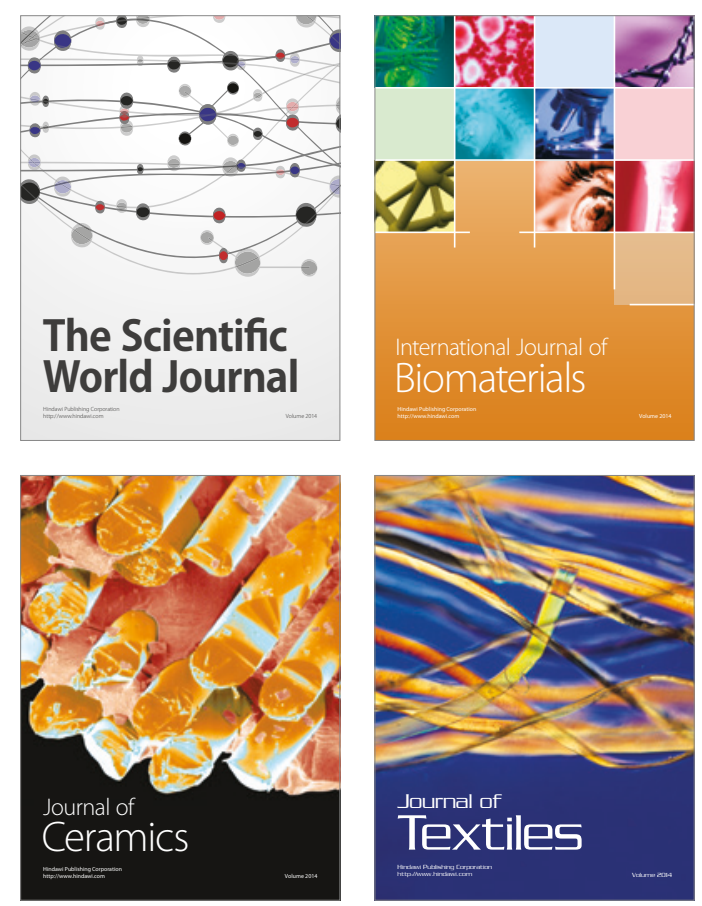